\title{
Anomalous Origin of the Left Coronary Artery from the Pulmonary Artery: A Review
}

\author{
Justin Fitch*, Christopher Ira Wertz and Trevor Mark Ward \\ Radiographic Science Program, Idaho State University, USA
}

Submission: March 29, 2019; Published: April 17, 2019

*Corresponding author: Justin Fitch, Radiographic Science Program, Idaho State University, 921 S. 8th Ave, Stop 8002, Pocatello, ID, 83209-8002, USA

\section{Abstract}

Anomalous origin of the left coronary artery from the pulmonary artery (ALCAPA) is a very rare but deadly congenital heart disease. If left untreated, patients usually die from a myocardial infarction in infancy or live to an average age of 35 . Detection of the disorder through the use of medical imaging such as X-ray, ultrasound, or coronary computed tomography angiography (CTA) is paramount to the survival of patients. X-ray and ultrasound are baseline procedures to detect ALCAPA. However, CTA is the optimal imaging modality to confirm ALCAPA because it provides the best detailed images in 3-D. Surgeons can diagnose, plan, and execute correctional procedures with higher confidence of CTA is used. A case report of a 31-year-old female with ALCAPA demonstrates the vital role of imaging for surgical correction to increase the chance to lead to a better prognosis.

Keywords: ALCAPA; Bland-White-Garland syndrome; X-ray; Computed Tomography Angiography; Ultrasound; Echocardiogram; myocardial infarction; Bypass grafting; Left coronary artery

Abbreviations: ALCAPA: Anomalous Origin of the Left Coronary Artery from the Pulmonary Artery; LCA: Left Coronary Artery; RCA: Right Coronary Artery, PA: Pulmonary Artery, TTE: Transthoracic Echocardiography; CTA: Computed Tomography Angiography, ECG: Electrocardiogram, LMCA: Left Main Coronary Artery; LADCA: Left Anterior Descending Coronary Artery

\section{Introduction}

Cardiovascular diseases are the primary cause of death worldwide. Even though coronary heart disease is responsible for the majority of deaths associated with the heart, there are many other less prevalent, but just as deadly, heart conditions [1]. Anomalous origin of the left coronary artery from the pulmonary artery (ALCAPA) is a rare heart anomaly [2-12]. ALCAPA is also known as Bland-White-Garland syndrome $[2,3,8,10]$. There are two types of ALCAPA: the infant-type and the adult-type."2(p.1010) Patients who have an inefficient intercoronary anastomoses with left coronary artery (LCA) arising from the pulmonary artery (PA) have infant-type ALCAPA. The adult-type have extensive intercoronary systems supplying the left coronary system with the LCA arising from the PA [ 2-3,8,10]. Both types have the LCA originating in the PA where oxygen-poor blood is located [ $2-3,8,10]$. Normally, the LCA originates in the aorta where there is oxygen enriched blood is present $[2-3,8,10]$. Because of this defect, the heart is not getting sufficient oxygen leading to the cardiac tissue to die causing the patient to have a heart attack [ $2-3,8,10]$. The purpose of this paper is to show the importance of medical imaging in the diagnosis and treatment of this rare and fatal medical disorder.

\section{Prevalence}

ALCAPA heart defect accounts for 0.25 percent to 0.5 percent of all heart abnormalities [2,4-5,7-8,10-11]. Infants have a very low chance of survival due to their vulnerability to congestive heart failure or myocardial ischemia. ALCAPA is the most common cause of heart attack in infants where 90 percent die in their first year of life unless they undergo surgery [5-8,11-12]. Only 10 to 15 percent of infants who do have the corrective surgery live past their first birthday however, but surgery is the child's best chance at survival $[4,6-8,11]$. Adults who have ALCAPA also need to have corrective surgery because it commonly leads to sudden death for 90 percent of them at an average age of 35 if left untreated $[4,6-8,11]$. Treatment is made possible with a higher level of success due to the availability of medical imaging such as chest x-rays, transthoracic echocardiography (TTE) utilizing color Doppler, coronary computed tomography angiography (CTA), and coronary angiography $[2-12,16]$. Without these modalities at the physician's disposal, the diagnosis and repair of ALCAPA would be non-existent.

\section{Anatomy and Physiology of the Heart}

The heart is located in the mediastinum enclosed in a doublewalled sac called the pericardium [13]. It is composed mainly of 
cardiac muscle which accounts for the majority of its size. Two superior atria and two inferior ventricles are the four chambers within the myocardium which handle the transport of the body's blood supply [13]. The interatrial septum separates the atria, and the interventricular septum separates the ventricles. Deoxygenated blood empties into the right atrium chamber after being dispersed throughout the body [13]. The blood is then pushed into the right ventricle where it will be pushed into the pulmonary arteries to get oxygen from the lungs. After becoming saturated in oxygen, the blood will empty into the left atrium [13]. Finally, the blood will go into the left ventricle which will contract to push the oxygenated blood into the aorta to supply the body with oxygen rich blood again $[2-3,8,10,13]$.

Even though the heart is continuously filled with blood, it does not obtain much nourishment because its myocardium is too thick for diffusion to work effectively [13]. To overcome this adversity, the heart has a coronary system arising from the aorta to provide it with the necessary nutrients it requires to perform its function [13]. The two main arteries nourishing the heart are the right and left coronary arteries [13]. The right coronary artery (RCA) supplies the right side of the heart, and the left coronary artery (LCA) supplies the left side. Both arteries anastomose to supply blood to the entire heart $[3,13]$. If either artery is occluded, then the cardiac tissue will begin to die leading to a myocardial infarction [3-6,8,11-13]. The heart represents a small part of the body, but it requires 5 percent of the body's blood supply to function [13].

Patients who have ALCAPA have the LCA originating in the PA, an underdeveloped coronary system, or an overdeveloped right coronary system compensating for an imperfect LCA [3]. The LCA should originate in the aorta where oxygen rich blood is present. It should not arise from the pulmonary artery where there is only oxygen-poor blood to supply the left coronary artery [3]. These anomalies lead to myocardial infarctions because oxygen-rich blood is not getting to all the portions of the heart to provide it with the necessary sustenance it needs to function and live [3].

\section{Signs and Symptoms of ALCAPA}

I. Signs and symptoms of ALCAPA are variable and depend on the patient's age [3]. Most cases of ALCAPA are in infants because ALCAPA is a congenital heart defect [2-12]. Some of the signs and symptoms the child could present with include [34,8,10-12,14-16]:
II. Dyspnea.
III. Change in physical color.
IV. Patent ductus arteriosus.
V. Moderate dilation of the left ventricle.
VI. Cardiomegaly.
VII. Myocardial ischemia.
VIII. Enlarged pulmonary artery

IX. Low ejection fractions such as 30 to 40 percent (Normal ranges are between 50 and 70 percent).

Adult patients diagnosed with ALCAPA have less severe symptoms when compared to children because they are still providing their heart with enough oxygenated blood despite the LCA originating in the PA. The most common signs and symptoms for both adults and children are angina and dyspnea [3-4,8,10-11,14-16]. It is important to recognize these signs and symptoms in patients so they can receive the necessary treatment. Early detection of ALCAPA is pivotal especially because of the progression of permanent ischemic tissue damage [8].

\section{ALCAPA Detection and Diagnoses}

ALCAPA is a very rare heart abnormality with an incidence rate of approximately 1 in 300,000 births, and it is only 0.24 percent to 0.46 percent of all congenital heart diseases [2]. This makes it very hard to find and diagnose. Today, the most frequently used imaging for ALCAPA are chest x-rays, transthoracic echocardiography (TTE) utilizing color Doppler, coronary computed tomography angiography (CTA), and coronary angiography $[2-12,16]$.

\section{Chest X-ray}

The least invasive means of examining the patient are usually used to determine the ailment. One of the least invasive and most prominent means of acquiring diagnostic imaging is $\mathrm{x}$-ray. A chest $\mathrm{x}$-ray is a common exam performed for a patient presenting with angina and dyspnea. The exam would rule out fluid accumulation in the lungs and it would show heart pathology. A patient with ALCAPA will usually show an enlarged heart shadow, mediastinal widening, and prominent upper lobe vessels $[8,10]$. An enlarged heart shadow is indicative of cardiomegaly and is an indication the heart is not circulating blood properly [10].

\section{Transthoracic echocardiography}

A transthoracic echocardiogram (TTE) uses ultrasound to evaluate the structures of the heart such as the chambers, valves, and vessels. TTE can also be used to measure ejection fraction in patients [2-12]. Those who have ejection fractions lower than 50 to 70 percent are at risk for ALCAPA. TTE is usually the first modality used by physicians when they suspect this anomaly. The most common signs seen from this procedure in ALCAPA patients are a low ejection fraction, and a dilation of the right coronary artery. The dilation is seen as a major indicator of a stenosis, obstruction, or inefficiency in the coronary system $[2-4,8,10$ 12,14-16]. Physicians typically utilize other imaging modalities to confirm the presence of ALCAPA from a TTE [2-4,8,10-12,14-16].

\section{Coronary computed tomography angiography and coronary angiography}

CTA is a noninvasive procedure with more benefits than $\mathrm{x}$-ray, ultrasound, and coronary angiography [2]. CTA produces detailed 3-D images of the vessels and their surroundings such as the intraluminal and extraluminal anatomy. CTA is performed employing a minimum 256 multidetector CT scanner [2]. The 
scan is done starting from the superior portion of the chest to the costophrenic angles to include the majority of the cardiovascular system [2]. Because the detector is able to do $256 \mathrm{~mm}$ by $0.625 \mathrm{~mm}$ width slices and have a pitch of 0.2 to 1 , high quality images are able to be obtained [2]. These are major contributors to prognostic information [2-5,8-10]. In the past, patients would undergo invasive coronary angiography following an ultrasound for suspicion of ALCAPA [2-6]. CTA allows surgeons to assess the illness without invasive angiography to make more definitive diagnoses with high quality images, make a blueprint for the corrective surgery, and perform the surgery in a more efficient manner to decrease the patient's risk and recovery time [2]. CTA should be the protocol in all heart abnormalities because it provides more detailed images of heart pathologies than x-ray or ultrasound, and it is less invasive than conventional coronary angiography.

\section{Case Report}

Saade et al. [2] depicted a 31-year-old female who presented to the emergency department with heart palpitations and progressive dyspnea. The patient had a healthy past history except for a mitral valve prolapse with regurgitation which was corrected 15 years earlier. She first underwent an electrocardiogram (ECG) which demonstrated atrial fibrillation cardioverted electrically with sinus rhythm. She was also experiencing habitual dyspnea on exertion without any symptoms of chest pain. The ECG then showed moderate dilation of her left ventricle and mild global left ventricle hypokinesia with an ejection fraction around 45 to 49 percent. During contraction, Doppler signals were demonstrated near the pulmonary valve which conveyed the origin of the left main coronary artery (LMCA) from the pulmonary artery [2].

Coronary CTA was used to confirm this deviation. The patient's right coronary artery (RCA) was prominent in the anterior mediastinum suggesting stenosis where the LMCA originates. The LMCA was originating from the inferior surface of the pulmonary artery and anastomosed with the right posterior descending coronary artery and the left anterior descending coronary artery (LADCA). After all of the imaging was accumulated and assessed, the patient elected to undergo surgery to reimplant the LMCA into the left coronary sinus in the ascending aorta. This method allows the oxygenated blood in the aorta to be supplied to the LMCA instead of the deoxygenated blood from the pulmonary artery [2].

\section{Treatments}

Treatments for ALCAPA are focused on reestablishing a dual coronary system with oxygenated blood being provided to the heart utilizing the least invasive and least risky procedure [2$4,8,10-12,14-16]$. The majority of the correctional procedures revolve around the origin of the LCA. If the artery is in proximity to the aorta, the preferred technique is the reimplantation method. However, if the reimplantation method cannot be performed, other alternatives for the patient include left coronary artery ligation, bypass grafting, and intrapulmonary tunneling, left coronary artery ligation, bypass grafting, or intrapulmonary tunneling which can be other alternatives for the patient [2-4]. These procedures are long lasting if performed correctly. They can be extremely effective and allow the heart to be available for transplant if necessary [15]

\section{Direct reimplantation}

Corrective surgery is a necessity for patients who have ALCAPA because of the high risk of myocardial ischemia and myocardial infarction, but an optimal technique has not been determined yet $[2-4,10-12,14-16]$. The most favored surgical option is direct reimplantation of the anomalous LCA from the pulmonary artery into the ascending aorta [2-4]. This method allows for the LCA to be inserted into the ascending aorta, an oxygen rich vessel, instead of the pulmonary artery which has deoxygenated blood [2-4]. The LCA is removed with a small portion of tissue from the pulmonary artery still attached to it, and it is implanted into the ascending aorta [2-4]. The heart's normal anatomy is restored coupled with a rebuilt dual coronary system. This method of correction is only viable if the patient's LCA is in close proximity to the ascending aorta. If the artery is not around the ascending aorta, then one of the other methods available must be employed $[2-4,8,10-12,14$ $16]$.

\section{Left coronary artery ligation and coronary artery bypass grafting}

One of the two other surgeries available to patients if a reimplantation of the LCA is not possible, is ligation of the LCA with coronary artery bypass grafting [2-4,8,14-16]. In this procedure, the surgeon blocks off or ligates the LCA [2-4,8]. After ligation, either an artery or vein from another part of the body is extracted and is used as a replacement vessel for the LCA. The new artery bypasses the nonfunctioning LCA and is attached into the aorta or another oxygen-rich vessel such as the subclavian artery. The other end of the artery is put into the section of heart in need of the oxygen-rich blood flow [2-4,8,10-12,14-16]. Ideally, the coronary arteries will anastomose to the new grafted artery to promote oxygen-rich blood flow. The procedure is not as preferred as the reimplantation method because there is higher chance of complications with ligation and bypass grafting of the LCA [2-4]. CTA is pivotal for both of these surgical corrections because it provides the detailed images necessary for the surgeons to plan out the patient's surgery [2-5,8-10].

\section{Intrapulmonary tunneling}

Another technique surgeons could use to resolve ALCAPA is intrapulmonary tunneling of the LCA in the pulmonary artery to the ascending aorta. The procedure is also known as the Tackeuchi operation [2-4,8,10-12]. This involves creating an anterior flap on the pulmonary artery and the ascending aorta allowing visible access to both the LCA and the aorta through the pulmonary artery [2-4,8,10-12]. The cardiologist essentially is forming a tunnel from the LCA to the aorta by means of the pulmonary artery instead of trying to transpose the LCA or bypassing the LCA [2-4,8,10-12]. The creation of this new baffle to the LCA allows for oxygen-rich blood from the aorta to pass through the LCA to supply its sections 
of the heart $[2-4,8,10-12]$. The procedure is very complex and has higher chances of risks than the reimplantation method, but it has about equal risk as the ligation and bypass grafting method [2$4,8,10-12]$.

\section{Conclusion}

ALCAPA is a very rare but very deadly ailment for all patient who are born with the anomaly. The condition is almost terminal because it most often leads to a myocardial infarction if surgical intervention is not performed. Physicians are very dependent on medical imaging to properly diagnose and treat patients who have ALCAPA. While noninvasive modalities such as $\mathrm{x}$-ray and ultrasound are usually performed first to look for pathologies, any heart abnormality should be imaged using noninvasive CTA to obtain he most detailed images of the pathology. The 3-D images convey more information than those of regular x-ray and ultrasound, and CTA provides a blueprint for surgeons to plan the best treatment for their patients. Because the disorder is congenital, the majority of patients are infants in need of immediate treatment. Other imaging besides CTA would prolong treatment and inhibit the physician from correcting the anomaly. Once ALCAPA is diagnosed and imaged, the disorder can be corrected with high success and allow the patient to live a normal life. Due to the low incidence of ALCAPA, more research is needed in the biology and cellular development of the infant to better understand the reason for the pathology's manifestation. The added research could lead to a reduction or elimination of the pathology saving infants from an invasive surgery.

\section{References}

1. Shi A, Tao Z, Wei P, Zhao J. Epidemiological aspects of heart diseases (Review). Exp Ther Med 12(3): 1645-1650.

2. Saade C, Al-Hamra S, Al-Mohiy H, El-Merhi F (2016) Contrast media delivery in the assessment of anomalous left coronary artery from the pulmonary artery. Radiol Technol 87(5): 490-494.

3. Aggarwal S, Delius RE, Pettersen MD (2013) Anomalous left coronary artery from the pulmonary artery with a large patent ductus arteriosus: Aversion of a catastrophe. Congenit Heart Dis 8(6): E168-E173.

4. Mungan U, Ozeke O, Mavioglu L, Sarisahin M, Ertan C, et al. (014) Adult type anomalous left coronary artery arising from the pulmonary ar- tery (ALCAPA): Complementary role of multimodality cardiac imaging. Herz 39(8): 1010-1012.

5. Formica F, Amigoni P, Mariani S, Coppadoro A, Mariani S, et al. (2014) A rare case of ALCAPA and rheumatic mitral valve regurgitation in an adult patient. Heart Surg Forum 17(5): E250-E252.

6. Talwar S, Jha AJ, Choudhary SK, Gupta SK, Airan B (2013) Repair of anomalous left coronary artery from pulmonary artery (ALCAPA) beyond infancy. Heart Surg Forum 16(4): E210-E215.

7. Ghaderi F, Gholoobi A, Moeinipour A (2014) Unique echocardiographic markers of anomalous origin of the left coronary artery from the pulmonary artery (ALCAPA) in the adult. Echocardiography 31(1): E13-E15.

8. Rodriguez-Gonzalez M, Tirado AM, Hosseinpour R, de Soto JS (2015) Anomalous origin of the left coronary artery from the pulmonary artery: Diagnoses and surgical results in 12 pediatric patients. Tex Heart Inst J 42(4): 350-356.

9. Day K, Avery R, Oliva I, Jokerst C (2014) Coronary CTA appearance of anomalous left coronary artery arising from the pulmonary artery with intramural aortic route. Int J Cardiol 30(2): 241-243.

10. Younus Z, Iftikhar R, Ahmed I (2013) Anomalous origin of left coronary artery from pulmonary artery (ALCAPA). J Coll Physicians Surg Pak 23(10): 743-744.

11. Quah JX, Hofmeyr L, Haqqani H, Clarke A, Rahman A, et al. (2014) The management of the older adult patient with anomalous left coronary artery from the pulmonary artery syndrome: A presentation of two cases and review of the literature. Congenit Heart Dis 9(6): E185-E194.

12. Kayıran PG, Kayıran SM, Gümüş T, Akçevin A, Dindar A (2013) Anomalous origin of left coronary artery from pulmonary artery (ALCAPA) in an infant with bronchiolitis and dilated cardiomyopathy. J Curr Pediatr 11(3): 142-145.

13. Marieb EN, Hoehn K (2015) Human Anatomy \& Physiology, (10 ${ }^{\text {th }}$ edn), Pearson Education Inc, San Francisco, CA, USA.

14. Hallbergson A, Rome JJ (2015) Percutaneous left main coronary artery stent for acute myocardial ischemia after repaired ALCAPA. Catheter Cardiovasc Interv 85(6): 1017-1020.

15. Simmonds JD, Mustafa M, Jaramillo DP, Bellsham-Revell HR, Marek J, et al. (2016) Successful orthotopic heart transplantation using a donor heart with ALCAPA. Pediatr Transplantation 20(6): 859-865.

16. Bobylev D, Meschenmoser L, Boethig D, Horke A (2015) Surgical repair of Shone's complex with anomalous origin of the left coronary artery arising from the right pulmonary artery. Interact Cardiovasc Thorac Surg 20(3): 439-442.

\section{Your next submission with Juniper Publishers will reach you the below assets}

- Quality Editorial service

- Swift Peer Review

- Reprints availability

- E-prints Service

- Manuscript Podcast for convenient understanding

- Global attainment for your research

- Manuscript accessibility in different formats

( Pdf, E-pub, Full Text, Audio)

- Unceasing customer service

Track the below URL for one-step submission

https://juniperpublishers.com/online-submission.php 